\title{
Multimode Synthesis Procedure For Microwave Filters Based On Thick Inductive Windows
}

\author{
M. Guglielmi, A. Alvarez and G. Gheri \\ European Space Research \\ and Technology Centre \\ Po. Box 299, Noordwijk, The Netherlands
}

\begin{abstract}
For several types of microwave filters for space application it is important to manufacture hardware without tuning elements. For this to be possible, one needs a systematic procedure to convert ideal elements, such as resonators and impedance inverters, into actual waveguide lengths and discontinuities. The situation is further complicated by the fact that waveguide discontinuities excite higher order modes that interacting with each other can have very strong effects. In this paper we first outline the theory behind a very efficient computer code for the simulation of microwave filters based on thick inductive windows. Then we describe in detail a step-by-step procedure that, based on the code developed, allows for the rapid design of this class of microwave filters without any tuning elements. Two actual examples of design are also discussed and comparisons presented between measurements and simulations.
\end{abstract}

\section{Introduction}

For several Communication-Satellite applications, it is of great importance to be able to manufacture microwave filters without any tuning elements. For this to be possible one needs a systematic procedure to convert ideal components, such as admittance or impedance inverters and lengths of transmission line [1], into actual hardware dimensions including all higher-order mode interactions. To this end, the equivalent network representation of waveguide discontinuities is a very useful tool.

Single-mode network representations have been derived for a large variety of structures [2], and have always been very popular with microwave engineers because they allow to represent a complex field problem in simple network form. The single-mode network representation is usually derived from an integral equation of known solution or from a variational expression. These network representations, however, are not accurate if there is significant higher-order mode interactions. The single-mode results have nevertheless been used extensively in the past, and the lack of ac- curacy of the models has been traditionally resolved with the introduction of tuning elements (usually screws) to be adjusted manually.

Recently, internal research work at the European Space Research and Technology Centre (ESTEC) has led to the development of a novel theoretical approach for the study of inductive steps in rectangular waveguide [3]. The theory developed is based on an integral equation formulation and gives as a result a multimode equivalent network representation (impedance matrix description) of the discontinuity that can rigorously account for all higher-order mode interactions. The key feature of this formulation is that the generic element of the impedance matrix depends only on the relative geometry of the problem and is essentially independent from frequency. This feature can be exploited very effectively to obtain computationally efficient codes.

In this paper we outline the theoretical formulation and its use in the implementation of a computer code for the analysis of rectangular waveguide filters based on thick inductive windows. In addition, we also describe in detail how the code produced can be used to effectively design waveguide filters starting from a given set of specifications. Two practical examples are discussed indicating how the theory developed can indeed lead to significant time savings in the manufacturing of this type of waveguide filter.

\section{Multimode Network Represen- tation}

The key element for the class of filters under investigation is the inductive step shown in Fig. 1. The excitation is chosen to be the fundamental $T E_{1,0}$ mode of the input rectangular waveguide so that only $T E_{m, 0}$ modes are excited in each waveguide. The details of the derivation of the multimode equivalent network representation for this discontinuity are reported in [3] and are not repeated here. The fundamental integral equation to be solved turns out to be 


$$
\begin{aligned}
& \sin \left(\frac{n \pi}{a^{(\gamma)}}\left(x-d^{(\gamma)}\right)\right)= \\
& \quad=\int_{d_{1}}^{d_{2}} M_{n}^{(\gamma)}\left(x^{\prime}\right) 2 B\left[\sum_{m=1}^{\infty} \frac{m \pi}{a^{(1)}} \sin \left(\frac{m \pi}{a^{(1)}} x\right) \sin \left(\frac{m \pi}{a^{(1)}} x^{\prime}\right)+\right. \\
& \left.\frac{a^{(1)}}{a^{(2)}} \sum_{m=1}^{\infty} \frac{m \pi}{a^{(2)}} \sin \left(\frac{m \pi}{a^{(2)}}\left(x-d^{(2)}\right)\right) \sin \left(\frac{m \pi}{a^{(2)}}\left(x^{\prime}-d^{(2)}\right)\right)\right] d x^{\prime}(1)
\end{aligned}
$$

where

$$
B=-\frac{j}{2 \omega \mu_{0}}
$$

and the expression for the multimode impedance coupling elements $z_{m, n}^{(\delta, \gamma)}$ is

$z_{m, n}^{(\delta, \gamma)}=\left(\frac{a^{(1)}}{a^{(2)}}\right)^{(\delta+\gamma-2) / 2} \int_{d_{2}}^{d_{1}} M_{n}^{(\gamma)}\left(x^{\prime}\right) \sin \left(\frac{m \pi}{a^{(\delta)}}\left(x^{\prime}-d^{(\delta)}\right)\right) d x^{\prime}$

Where $\delta$ and $\gamma$ are equal to 1 or 2 indicating the guide to the left or the one to the right of the step, respectively. The solution of the integral equation is not known in closed form so that a method-of-moment procedure is adopted [4].

Combining together a series of equivalent network representations of individual steps one can develop the complete multimode equivalent network representation of a multicavity filter, as shown in Fig. 2 for the simpler case of a single cavity. From this equivalent network representation one can easily derive a linear system of equation that, with one matrix inversion per frequency point, gives the overall electrical behavior of the structure.

The code developed following this approach is very efficient computationally for two main reasons. The first is that the kernel of (1) is frequency independent so that all step discontinuities considered need only be characterized once for each given structure. The second is that the expression for the coupling matrix element in (3) only depends on the relative step geometry. As a result of this last point, the integral equation (1) needs only be solved for a discrete set of relative step values (for instance 20 ) and all other intermediate values can then be computed via a simple linear interpolation procedure without using the complete formulation. These unique features of the formulation result in a code implementation that is very efficient both in terms of speed and memory occupation.

\section{Filter Synthesis}

The first step in the design of a microwave filter is the selection of the proper transfer function and relative low-pass or band-pass equivalent network. For the case of Tchebyscheff filters one can use, for instance, the results given in [1] that relate the filter transfer function to proper resonance frequencies, values of external $\mathrm{Q}$-factor $Q_{e}$ and inter-resonator couplings $k_{j, j+1}$, using the following well-known expressions

$$
Q_{e}=\frac{g_{o} g_{1}}{w}
$$

$$
k_{j, j+1}=\frac{w}{\sqrt{g_{j} \bar{g}_{j+1}}}
$$

where $w$ is the fractional bandwidth of the filter and the coefficient $g_{j}$ are tabulated in [1]. Following this formalism, the problem is reduced to finding the coupling-aperture widths to obtain the proper values of $Q_{e}$ and $k_{j, j+1}$, and the resonator lengths corresponding to the design center frequency. The difficulty in obtaining the correct values is in that the various coupling apertures interact with each other trough the higher-order modes of the waveguide.

The CAD package developed following the theory described in the previous section can be used to generate design curves to select the initial set of geometrical parameters. The procedure is very simple. We first select the thickness of the windows (2mm for instance) and an appropriate length of waveguide to give a $\lambda_{g} / 2$ resonator at a frequency slightly higher that the design center frequency (10\% for instance). Then we perform a set of "software" (soft) experiments on doubly-terminated single and double cavities to obtain curves similar to the ones in Figs. 3 and 4 for $Q_{e}, k_{j, j+1}$ and the actual resonance frequency $f_{m}$, respectively, versus the relative aperture width $a_{m} / \lambda_{g m}$.

It is important to note that in order to obtain accurate results, a number of higher-order modes must be explicitly included in the computations performed. We have found that at least 6 modes in each waveguide region must be included to ensure the proper convergence. All results presented in this paper have been obtained explicitly including 10 modes in each waveguide region.

The next step consists of the utilization of the curve for $f_{m}$ vs $a_{m} / \lambda_{g m}$ to obtain the curves in Fig. 5 representing the apparent waveguide-length increase $\Delta l_{m} / \lambda_{g m}$

$$
\frac{\Delta l_{m}}{\lambda_{g m}}=0.5\left(1-\frac{\lambda_{g}}{\lambda_{g m}}\right)
$$

due to the loading introduced by the coupling apertures. The data for the curves in Fig. 3 and 4 are obtained by using the code developed, to perform the "soft" experiments, and the well-known equations

$$
\begin{gathered}
Q_{e}=2 \frac{f_{m}}{\Delta f_{3 d B}} \\
k_{1,2}=\sqrt{w_{m}^{2}+\left(\frac{1}{Q_{e}}\right)^{2}}
\end{gathered}
$$

where $f_{m}$ is the resonance frequency and $\Delta f_{3 d B}$ is the $3 d B$ bandwidth of the single resonator. The parameter $w_{m}$ is given by

$$
w_{m}=\frac{f_{2}-f_{1}}{f_{m}}
$$

where $f_{2}$ and $f_{1}$ are the upper and lower frequencies corresponding to the resonant "dips" in the magnitude of the $S_{11}$ parameter of an overcoupled pair of resonators, and $f_{m}$ is the center frequency of the pair of resonators. 
The results presented have been obtained by actually computing only a discrete set of points (indicated by the stars). The smooth curves have then been obtained with polynomial interpolation, namely

$$
y=\sum_{n=0}^{N} a_{n} x^{n}
$$

The initial selection of the geometrical parameters can now proceed by first computing the length of waveguide $l_{d}$ that would give a $\lambda_{g d} / 2$ resonator at the design frequency $f_{d}$. The second step is the use the following novel expression

$$
a_{d}=\frac{a_{m}}{\lambda_{g m}} \lambda_{g d} \frac{f_{d}}{f_{m}}
$$

to obtain the apertures corresponding to the desired $Q_{e}$ and the $k_{j, j+1}$ values. In all equations presented, the subscript $m$ indicates values from the design curves while the subscript $d$ indicates the required design value.

Finally the individual length of the resonators is adjusted using the information from Fig. 5 and the expression

$$
\Delta l_{d}=\frac{\Delta l_{m}}{\lambda_{g m}} \lambda_{g d}
$$

so that the design length $l_{d, j}$ is given by

$$
l_{d, j}=\frac{\lambda_{g d}}{2}-\Delta l_{d, j}-\Delta l_{d, j+1}
$$

where $\Delta l_{d, j}$ and $\Delta l_{d, j+1}$ refer to the contributions of the two coupling apertures seen by each cavity. This process is repeated until all needed apertures and resonator lengths are obtained thus completing the initial choice of the geometrical parameters.

It is important to observe that, although the above expressions have been derived empirically, they are nevertheless extremely useful. In fact, their use results in a filter structure that can be optimized by an experienced engineer to meet the design goal with only a few manual "soft" tunings. All the design formulas presented are exact only if the design and measurement frequency coincide. As the distance between measurement and design increases, their accuracy decreases. They are, however, very usefull in that they provide a very good starting point, as will be discussed in the next section.

\section{Application examples}

To fully appreciate the power of the CAD procedure, we describe in this section the design of two filters. Figure 6 gives the physical dimensions of the filters as obtained from the design procedure described in the previous section. Also in Fig. 6 we give the final dimensions of the filters after manual "soft" tuning. It is important to note that the best results from this design procedure are obtained when the measurement frequency is higher than the design frequency by about $10 \%$. In the two cases discussed, however, the design frequency is actually lower by about $15 \%$. As we can see from Fig. 6, even in this case, the procedure outlined provides a good starting point from which the desired final result can be obtained with little effort.

To verify the theory developed, both filters have been realized using spark erosion. In Figure 7 we present the simulated and computed electrical characteristics of the filters. As we can see, the agreement between measure and simulation is indeed very good.

\section{Conclusion}

In this paper we outline the theory behind a CAD package for the design of microwave filters based on thick inductive windows in rectangular waveguide. We describe a step-by-step procedure that, based on the use of code developed, allows to select very accurately the initial geometry of the filter. Finally, we give two filter-design examples that demonstrate the effectiveness of the CAD procedure developed.

The procedure described is in essence a true CAD technique that can completely replace all of the hardware work and all of the manual tunings in the laboratory that are traditionally required for the manufacture of this class of waveguide filters thus resulting in a significant reduction in development time and cost.

\section{References}

[1] G. Matthaei, L. Young, E. M. T. Yones, Microwave Filters, Impedance-Matching Networks, and Coupling Structures, ARTECH HOUSE BOOKS, Denham, Massachussetts, 1980.

[2] N. Marcuvitz, Waveguide Handbook (MIT Radiation Laboratory Series, Vol. 10) McGraw-Hill Book Co., New York, 1951.

[3] M. Guglielmi and G. Gheri, "Rigorous Multimode Network/Numerical Representation of Inductive Step," Accepted for publication in the IEEE Microwave Theory Tech, July 1992.

[4] T. Itoh, Numerical Techniques for Microwave and Millimeter-Wave Passive Structures, John Wiley and Sons INC., New York, 1989.

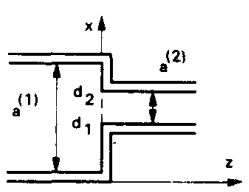

Fig. 1 Top view of inductive step in rectangular waveguide. 

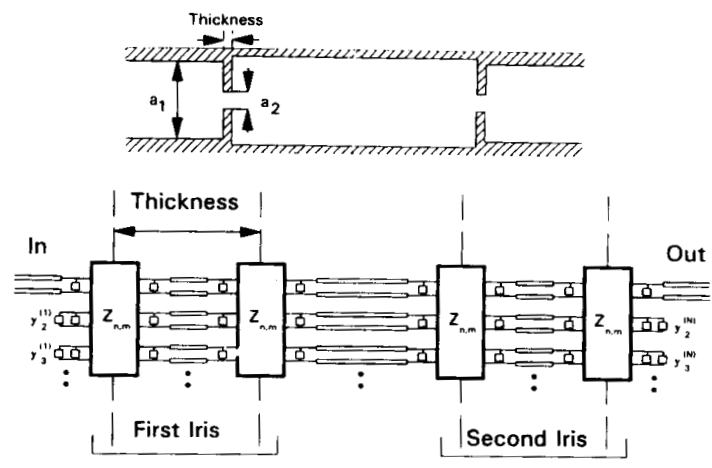

Fig. 2 Multimode equivalent network representation of a single cavity.
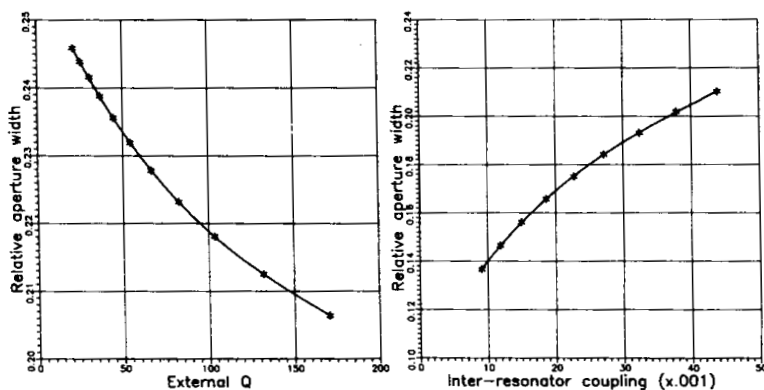

Fig. 3 External quality factor $Q_{e}$ for a single doublyterminated cavity and inter resonator coupling $k_{1,2}$ for a pair of resonators in doubly terminated configuration as a function of the relative aperture $a_{m} / \lambda_{g m}$.
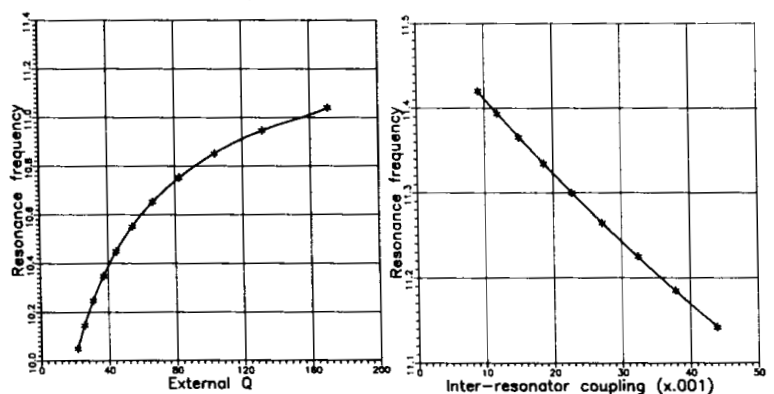

Fig. 4 Effective resonance frequency $f_{m}$ of single and coupled resonators as a function of the relative aperture $a_{m} / \lambda_{g m}$ for doubly-terminated configuration.
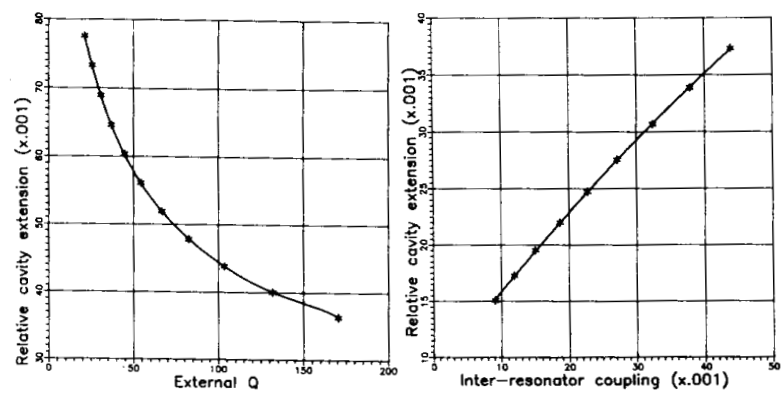

Fig. 5 Apparent cavity-length increase $\Delta l_{m} / \lambda_{g m}$ due to the coupling-aperture loading.
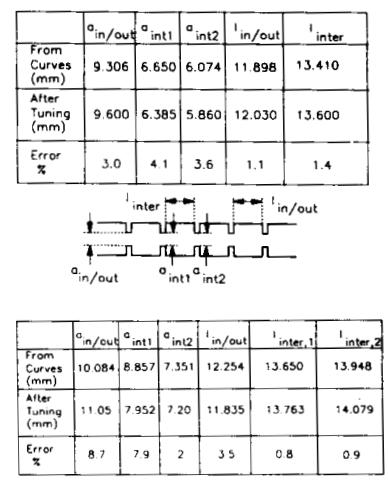

Fig. 6 Physical dimensions of the filters designed using the approach described. Both initial dimensions (from CAD procedure) and final dimensions (after "soft" tuning) are given.
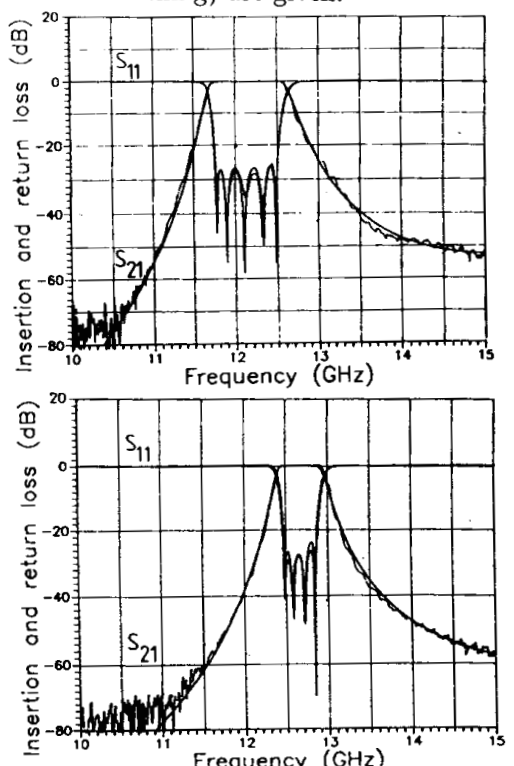

Fig. 7 Simulated and measured performance. The smooth line represents the simulation. 